\title{
Electrohydrodynamics of binary electrolytes driven by modulated surface potentials
}

\author{
Mortensen, Asger; Olesen, Laurits Højgaard; Belmon, L.; Bruus, Henrik
}

Published in:

Physical Review E

Link to article, DOI:

10.1103/PhysRevE.71.056306

Publication date:

2005

Document Version

Publisher's PDF, also known as Version of record

Link back to DTU Orbit

Citation (APA):

Mortensen, A., Olesen, L. H., Belmon, L., \& Bruus, H. (2005). Electrohydrodynamics of binary electrolytes driven by modulated surface potentials. Physical Review E, 71(5), 056306.

https://doi.org/10.1103/PhysRevE.71.056306

\section{General rights}

Copyright and moral rights for the publications made accessible in the public portal are retained by the authors and/or other copyright owners and it is a condition of accessing publications that users recognise and abide by the legal requirements associated with these rights.

- Users may download and print one copy of any publication from the public portal for the purpose of private study or research.

- You may not further distribute the material or use it for any profit-making activity or commercial gain

- You may freely distribute the URL identifying the publication in the public portal

If you believe that this document breaches copyright please contact us providing details, and we will remove access to the work immediately and investigate your claim 


\title{
Electrohydrodynamics of binary electrolytes driven by modulated surface potentials
}

\author{
Niels Asger Mortensen, ${ }^{1}$ Laurits Højgaard Olesen, ${ }^{1}$ Lionel Belmon, ${ }^{1,2}$ and Henrik Bruus ${ }^{1}$ \\ ${ }^{1}$ MIC_Department of Micro and Nanotechnology, Technical University of Denmark, DK-2800 Kongens Lyngby, Denmark \\ ${ }^{2}$ Ecole Centrale de Nantes, F-44321 Nantes, France
}

(Received 7 July 2004; revised manuscript received 14 December 2004; published 24 May 2005)

\begin{abstract}
We study the electrohydrodynamics of the Debye screening layer that arises in an aqueous binary solution near a planar insulating wall when applying a spatially modulated ac voltage. Combining this with first order perturbation theory we establish the governing equations for the full nonequilibrium problem and obtain analytic solutions in the bulk for the pressure and velocity fields of the electrolyte and for the electric potential. We find good agreement between the numerics of the full problem and the analytics of the linear theory. Our work provides the theoretical foundations of circuit models discussed in the literature. The nonequilibrium approach also reveals unexpected high-frequency dynamics not predicted by circuit models.
\end{abstract}

DOI: 10.1103/PhysRevE.71.056306

PACS number(s): 47.65.+a, 47.32. $-\mathrm{y}, 47.70 .-\mathrm{n}, 85.90 .+\mathrm{h}$

\section{INTRODUCTION}

Recently, there has been quite some interest in electrohydrodynamics in microfluidic systems. ac-driven, modulated surface potentials have been used for pumping, fluid circulation, and mixing [1-13]. For an overview of ac electroosmosis we refer to Refs. [14-19] and references therein.

Although ac electro-osmosis is typically analyzed with the help of homogeneous circuit elements open questions remain about the applicability of such approaches [19]. We reconsider the problem studied by Ajdari [2] where an electrolyte is perturbed by an ac-driven spatially modulated surface potential, but include explicitly an insulating layer between the electrode providing the driving potential and the electrolyte. We primarily think of this insulator as an oxide grown intentionally for device purposes, but it could also represent the molecular Stern layer in case of nonoxidized electrodes. We develop a full nonequilibrium description of the electrohydrodynamics of this system thus extending previous modeling of the surface and the Debye layer as simple capacitors. This allows us to study the full dynamics of ion concentrations, electrical potentials, velocity fields, pressure gradient fields, and electrical currents as well as the justifications for a description based on homogeneous circuit elements.

In the following we consider a binary electrolyte, i.e., an aqueous solution of a salt containing a positive and a negative type of ions with charges $+Z e$ and $-Z e$, respectively, where $Z$ is the valence and $e$ the elementary charge. In terms of Cartesian coordinates $x y z$ the electrolyte is confined to the semi-infinite space $x>0$ by an impenetrable, homogeneous, and planar insulating layer with dielectric constant $\epsilon_{s}$ placed at $-d<x<0$ (see Fig. 1). The insulating layer is bounded by a conductor at $x<-d$ which has been biased at the surface $x=-d$ by a spatially modulated, external ac potential $V_{\text {ext }}(y, t)$,

$$
V_{\text {ext }}(y, t)=V_{0} \cos (q y) e^{i \omega t},
$$

where $V_{0}$ is the amplitude, $q$ the wave number of the spatial modulation, and $\omega$ the driving angular frequency.
There is complete translation invariance along the $z$ axis, so the $z$ coordinate drops out of our analysis, and all positions $\mathbf{r}=x \mathbf{e}_{x}+y \mathbf{e}_{y}$ are therefore just referring to the $x y$ plane.

The paper is organized as follows. In Sec. II we present the nonequilibrium description and in Secs. III and IV we analytically study linearized equations of the static and dynamic regimes, respectively. In Sec. V we study numerical solutions of the fully coupled nonlinear electrohydrodynamic problem. Finally, in Sec. VI we compare these solutions with the analytical solutions of the linearized equations, and furthermore contrast our results with the literature, before we conclude in Sec. VII.

\section{NONEQUILIBRIUM DESCRIPTION}

The basic nonequilibrium formalism for continuum electrohydrodynamics is well known (see, e.g., Ref. [19]), but as mentioned in the Introduction we explicitly include an insulating layer in the description. We do not include an intrinsic $\zeta$ potential, i.e., no unpassivated surface charges on the insulator-electrolyte interface. We note that experimentally any intrinsic homogeneous $\zeta$ potential may be compensated

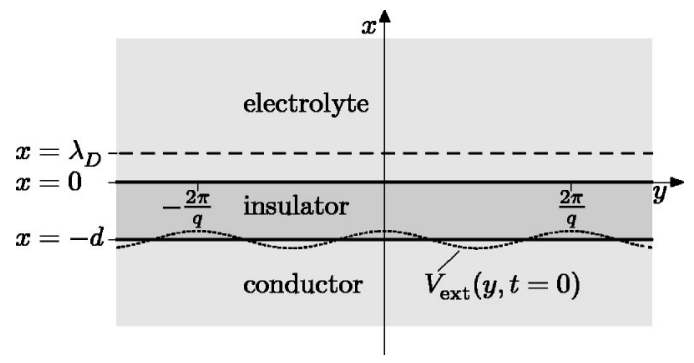

FIG. 1. A sketch of the system under study. The binary electrolyte is situated in the half space $x>0$. Below it, for $-d<x<0$, is a planar wall consisting of an insulating dielectric slab of thickness $d$ and below that, for $x<-d$, is a semi-infinite conductor. The top surface, $x=-d$, of the conductor is biased by a periodically modulated potential $V_{\text {ext }}(y, t)$ of period $2 \pi / q$ (dotted line), which gives rise to the formation of a Debye screening layer of thickness $\lambda_{D}$ in the electrolyte (dashed line). 
TABLE I. Typical values of central parameters.

\begin{tabular}{lcc} 
Spatial modulation & $q^{-1}$ & $10^{-5} \mathrm{~m}$ \\
Insulator thickness & $d$ & $10^{-8} \mathrm{~m}$ \\
Debye length & $\lambda_{D}$ & $10^{-8} \mathrm{~m}$ \\
Resonance frequency & $\omega^{*}$ & $10^{5} \mathrm{rad} / \mathrm{s}$ \\
Debye frequency & $\omega_{D}=\sigma_{\infty} / \epsilon$ & $10^{7} \mathrm{rad} / \mathrm{s}$ \\
Critical frequency & $\omega_{c}=(\eta / \rho) q^{2}$ & $10^{4} \mathrm{rad} / \mathrm{s}$ \\
Thermal voltage & $V_{T}=(1+\delta) k_{\mathrm{B}} T / Z e$ & $250 \mathrm{mV}$ \\
Convective voltage & $V_{c}=\sqrt{(1+\delta) \eta D / \epsilon}$ & $100 \mathrm{mV}$ \\
Ionic density & $n_{\infty}$ & $1 \mathrm{~mol} \mathrm{l}^{-1}$ \\
Viscosity & $\eta$ & $10^{-3} \mathrm{~Pa} \mathrm{~s}$ \\
Mass density & $\rho$ & $10^{3} \mathrm{~kg} \mathrm{~m}^{-3}$ \\
Ionic diffusivity & $D$ & $10^{-9} \mathrm{~m}^{2} \mathrm{~s}^{-1}$ \\
Capacitance ratio & $\delta=C_{D} / C_{s}$ & 10 \\
\hline \hline
\end{tabular}

by a corresponding dc shift added to the applied ac potential. Due to heterogeneous surfaces, it may be anticipated that although the average $\zeta$ potential is nulled out, there might be fluctuations left. These will be the topic for future work. For a zero intrinsic $\zeta$ potential we solve the full nonlinear equations numerically, but to obtain analytical results we also study the linearized equation with special emphasis on the capacitance due to the insulating layer. In Sec. V we show that for experimental relevant parameters, the linear theory is surprisingly good. Typical values of various central parameters are listed in Table I.

\section{A. The insulating layer, $-d<x<0$}

The insulating layer contains neither free space charge nor free currents so the electrical potential $\phi(\mathbf{r}, t)$ is governed by the Laplace equation,

$$
\nabla^{2} \phi(\mathbf{r}, t)=0 \text { for }-d<x<0 .
$$

\section{B. The electrolyte, $x>0$}

In the liquid electrolyte we consider the ionic densities $n^{ \pm}(\mathbf{r}, t)$, the potential $\phi(\mathbf{r}, t)$, the ionic current densities (the ionic flux densities) $\mathbf{i}^{ \pm}(\mathbf{r}, t)$, the velocity field $\mathbf{v}(\mathbf{r}, t)$ of the electrolyte, and the pressure $p(\mathbf{r}, t)$. In the following we suppress $(\mathbf{r}, t)$ unless needed for clarity.

The number densities of the ions couple to the potential via Poisson's equation,

$$
\nabla^{2} \phi=-\frac{Z e}{\epsilon}\left(n^{+}-n^{-}\right)
$$

The ionic current densities are coupled to the ionic densities by a continuity equation, which in the absence of any chemical reactions in the system is

$$
\partial_{t} n^{ \pm}=-\nabla \cdot \mathbf{i}^{ \pm} .
$$

The presence of convection or of gradients in the densities $n^{ \pm}$and the electric potential $\phi$ will generate ionic current densities $\mathbf{i}^{ \pm}$. The Nernst-Planck equation gives these currents

$$
\mathbf{i}^{ \pm}=-D \nabla n^{ \pm}+n^{ \pm} \mathbf{v} \mp \mu n^{ \pm} \nabla \phi,
$$

where, for simplicity, we have assumed that the two types of ions have the same diffusivity $D$ and the same mobility $\mu$. We remind the reader that both the diffusivity $D$ and the electric conductivity $\sigma$ are linked to the mobility $\mu$ via the Einstein relation $D=\left(k_{\mathrm{B}} T / Z e\right) \mu$ and $\sigma^{ \pm}=Z e n^{ \pm} \mu$.

Finally, the velocity field and pressure of the liquid are coupled to the potential and ionic densities by the NavierStokes equation

$$
\rho\left[\partial_{t} \mathbf{v}+(\mathbf{v} \cdot \boldsymbol{\nabla}) \mathbf{v}\right]=-\boldsymbol{\nabla} p+\eta \boldsymbol{\nabla}^{2} \mathbf{v}-Z e\left[n^{+}-n^{-}\right] \boldsymbol{\nabla} \phi,
$$

where $\rho$ is the mass density, $\eta$ is the viscosity of the liquid, and $p$ is the pressure. Furthermore, treating the electrolyte as an incompressible fluid we have

$$
\boldsymbol{\nabla} \cdot \mathbf{v}=0 \text {. }
$$

The coupled field equations Eqs. (3a)-(3e) fully govern the physical fields $n^{ \pm}, \phi, \mathbf{i}^{ \pm}, \mathbf{v}$, and $p$.

\section{Boundary conditions}

Assuming a vanishing $\zeta$ potential the boundary condition for the electric potential is

$$
\begin{gathered}
\left.\phi(\mathbf{r}, t)\right|_{x=-d}=V_{\text {ext }}(y, t), \\
\left.\phi(\mathbf{r}, t)\right|_{x=\infty}=0 .
\end{gathered}
$$

At the interface between the electrolyte and the insulating region the normal component of the ionic current density vanishes,

$$
0=\left.\partial_{x} n^{ \pm}(\mathbf{r}, t)\right|_{x=0} \pm\left.\frac{Z e}{k_{\mathrm{B}} T} n^{ \pm}(\mathbf{r}, t) \partial_{x} \phi(\mathbf{r}, t)\right|_{x=0} .
$$

Here, we have utilized Eq. (3c) and the absence of convection at the interface due to the no-slip boundary condition,

$$
\left.\mathbf{v}(\mathbf{r}, t)\right|_{x=0}=\mathbf{0} .
$$

For the ionic densities we have

$$
\left.n^{ \pm}(\mathbf{r}, t)\right|_{x=\infty}=n_{\infty},
$$

where $n_{\infty}$ is the homogeneous density of either of the two types of ions in the absence of an external perturbation, i.e., when $V_{0}=0$. For the pressure, we assume that we have no externally applied pressure gradients so that $p$ is the internal pressure caused by fluid flow and the electrical forces on the ions.

\section{STATIC REGIME, $\omega=0$}

In the static regime we have equilibrium and neither current nor fluid flow, i.e., $\mathbf{i}^{ \pm}=\mathbf{0}$ and $\mathbf{v}=\mathbf{0}$. The pressure gradient balances the electrical forces on the charges. The governing equations for $\phi$ and $n^{ \pm}$of course reduce to those of electrostatics. 

that

In the insulating layer it follows from Eqs. (2) and (4a)

$$
\phi(\mathbf{r})=\left[\mathcal{B}_{1} e^{-q x}+\mathcal{B}_{2} e^{q x}\right] \cos (q y) \text { for }-d<x<0,
$$

where $\mathcal{B}_{1}$ and $\mathcal{B}_{2}$ are integration constants.

In the electrolyte $\phi(\mathbf{r})$ is governed by the nonlinear Poisson-Boltzmann equation [19]

$$
\nabla^{2} \phi(\mathbf{r})=\frac{k_{\mathrm{B}} T}{Z e \lambda_{D}^{2}} \sinh \left[\frac{Z e}{k_{\mathrm{B}} T} \phi(\mathbf{r})\right] \text { for } x>0,
$$

introducing the Debye screening length

$$
\lambda_{D} \equiv \sqrt{\frac{\epsilon k_{\mathrm{B}} T}{2 Z^{2} e^{2} n_{\infty}}} .
$$

For $q$ going to zero we have a constant surface potential

$$
\left.\lim _{q \rightarrow 0} \phi(\mathbf{r})\right|_{x=0} \equiv \phi_{0}
$$

and the solution to Eq. (9) is given by the well-known GouyChapman solution [19]

$$
\lim _{q \rightarrow 0} \phi(\mathbf{r})=\frac{4 k_{\mathrm{B}} T}{Z e} \operatorname{arctanh}\left[\tanh \left(\frac{Z e \phi_{0}}{4 k_{\mathrm{B}} T}\right) e^{-x / \lambda_{D}}\right] .
$$

For $q \neq 0$ we are not aware of any analytical solutions, but as we shall show, analytical results can be obtained in the Debye-Hückel approximation $Z e \phi \ll k_{\mathrm{B}} T$, where Eq. (9) becomes linear,

$$
\nabla^{2} \phi(\mathbf{r})=\lambda_{D}^{-2} \phi(\mathbf{r}) \quad \text { for } x>0 .
$$

Here, the corrections are to third order in $Z e \phi / k_{\mathrm{B}} T$ because $\sinh (x)=x+O\left(x^{3}\right)$. The space charge follows from Poisson's equation Eq. (3a). From a straightforward solution for $\phi$ and $\mathrm{Ze}\left(n^{+}-n^{-}\right)$we arrive at the following expression relating the total potential drop across the system and the accumulated charge in the electrolyte:

$$
\phi(\infty, y)-\phi(-d, y) \equiv C_{\text {eff }}^{-1} \int_{0}^{\infty} d x Z e\left[n^{+}(\mathbf{r})-n^{-}(\mathbf{r})\right] .
$$

The coefficient

$$
C_{\text {eff }}^{-1}=\left[1+\left(q \lambda_{D}\right)^{2}\right] \frac{\sinh (q d)}{q d} C_{s}^{-1}+\sqrt{1+\left(q \lambda_{D}\right)^{2}} \cosh (q d) C_{D}^{-1}
$$

is identified as the inverse of an effective series capacitance. The constant $C_{s}$ is the intrinsic surface capacitance and $C_{D}$ the capacitance of the Debye layer given by

$$
\begin{gathered}
C_{s} \equiv \frac{\epsilon_{s}}{d}, \\
C_{D} \equiv \frac{\epsilon}{\lambda_{D}} .
\end{gathered}
$$

In Ref. [2] the potential in the bulk of the electrolyte ( $x$ $\gg \lambda_{D}$ ) is governed by the Laplace equation, which is coupled to the external potential $V_{\text {ext }}$ by an effective capacitance $C_{0}$ given by

$$
C_{0} \equiv\left(C_{s}^{-1}+C_{D}^{-1}\right)^{-1} .
$$

It follows from Eq. (13b) that this approach for $\omega=0$ is valid up to second order in the parameters $q \lambda_{D}$ and $q d$.

\section{LINEARIZED DYNAMIC REGIME, $\omega>0$}

We now solve Eqs. (3a)-(3e) in the dynamic regime, $\omega$ $>0$. First the ionic current densities are eliminated by inserting Eq. (3c) into Eq. (3b). Using the incompressibility of the fluid, Eq. (3e), we get the continuity equation

$$
\partial_{t} n^{ \pm}=D \boldsymbol{\nabla}^{2} n^{ \pm}-\left(\boldsymbol{\nabla} n^{ \pm}\right) \cdot \mathbf{v} \pm \mu \boldsymbol{\nabla} \cdot\left(n^{ \pm} \boldsymbol{\nabla} \phi\right) .
$$

\section{A. Debye-Hückel approximation}

To advance further by analytical methods, we now linearize the continuity equation Eq. (14) in the density as follows. We assume $\left.n^{ \pm}(\mathbf{r}, t)\right|_{x=\infty} \equiv n_{\infty}$ and write

$$
n^{ \pm}(\mathbf{r}, t)=n_{\infty}+\delta n^{ \pm}(\mathbf{r}, t), \quad \lim _{x \rightarrow \infty} \delta n^{ \pm}(\mathbf{r}, t)=0 .
$$

Since we assume a zero intrinsic $\zeta$ potential it is a nonzero $V_{0}$ that spawns $\delta n^{ \pm} \neq 0$, and when the applied voltage $V_{0}$ is much smaller than the thermal voltage $V_{T}$, defined by $V_{T}$ $\equiv\left(1+C_{D} / C_{S}\right) k_{\mathrm{B}} T / Z e$ (as we shall see in the next subsection), we have $\left|\delta n^{ \pm}\right| \ll n_{\infty}$. In this limit the Debye-Hückel approximation is valid, and $n^{ \pm} \nabla \phi$ is substituted by $n_{\infty} \boldsymbol{\nabla} \phi$ in Eq. (14). We subsequently use Eq. (3a) to replace $\nabla^{2} \phi$ with $-Z e \nu / \epsilon$ where

$$
\nu \equiv n^{+}-n^{-}=\delta n^{+}-\delta n^{-} .
$$

Finally, we form the difference of the \pm versions of Eq. (14) and obtain the partial differential equation

$$
\partial_{t} \nu=\left[D \nabla^{2}-D \frac{1}{\lambda_{D}^{2}}-\mathbf{v} \cdot \boldsymbol{\nabla}\right] \nu .
$$

\section{B. Diffusive regime}

Our study of the static regime reconfirms the well-known result that the net charge density is nonzero only in the Debye layer, $x \leq 3 \lambda_{D}$. In this region convection will be suppressed due to the no-slip boundary condition. Thus, convection can be neglected, diffusion will dominate (corresponding to a low Péclet number), and the electrodynamics can be solved independently of the hydrodynamics. On the other hand, the hydrodynamics of course still depends on the electrodynamics via the body force. Since the density difference $\nu$ changes over the length scales $\lambda_{D}$ and $q^{-1}$ for the $x$ and $y$ directions, respectively, the condition for the decoupling is $\left|v_{x}\right| / \lambda_{D}+\left|v_{y}\right| q \ll D q^{2}$ for $0<x \leqq 3 \lambda_{D}$. In this limit Eq. (17) has a general $\cos (q y) e^{i \omega t}$ modulated decaying solution of the form

$$
\nu=\mathcal{C}_{1} e^{-\kappa x} \cos (q y) e^{i \omega t}, \quad x>0,
$$

where the decay parameter $\kappa$ depends on the ratio between the frequency $\omega$ and the Debye frequency $\omega_{D}$, 


$$
\begin{gathered}
\kappa \equiv \frac{1}{\lambda_{D}} \sqrt{1+\left(q \lambda_{D}\right)^{2}+i \frac{\omega}{\omega_{D}}}, \\
\omega_{D} \equiv \frac{D}{\lambda_{D}^{2}} .
\end{gathered}
$$

For the potential we seek a solution of a form similar to Eq. (18a), $\phi \propto \cos (q y) e^{i \omega t}$, and substituting this together with Eq. (18a) into Eq. (3a) yields

$$
\left(\partial_{x}^{2}-q^{2}\right) \phi=-\frac{Z e}{\epsilon} \mathcal{C}_{1} e^{-\kappa x} \cos (q y) e^{i \omega t} .
$$

Demanding $\left.\phi(\mathbf{r}, t)\right|_{x=\infty}=0$ the solution is

$$
\phi=\frac{Z e / \epsilon}{q^{2}-\kappa^{2}}\left[\mathcal{C}_{1} e^{-\kappa x}+\mathcal{C}_{2} e^{-q x}\right] \cos (q y) e^{i \omega t}, \quad x>0
$$

In the insulating layer we have the following $\cos (q y) e^{i \omega t}$ modulated general solution to Eq. (2):

$$
\phi=\left[\mathcal{C}_{3} e^{-q x}+\mathcal{C}_{4} e^{q x}\right] \cos (q y) e^{i \omega t}, \quad-d<x<0 .
$$

In order to determine $\mathcal{C}_{1}, \mathcal{C}_{2}, \mathcal{C}_{3}$, and $\mathcal{C}_{4}$ we first consider the boundary condition for the current. Applying the DebyeHückel approximation to the second term in Eq. (5) and forming the difference of the \pm solutions we arrive at

$$
0=\left.\partial_{x}\left[\nu(\mathbf{r}, t)+\frac{C_{D}}{Z e \lambda_{D}} \phi(\mathbf{r}, t)\right]\right|_{x=0} .
$$

Together with the boundary condition for $\phi$ at $x=-d$ as well as the continuity of $\phi$ and $\epsilon \partial_{x} \phi$ at $x=0$ we may determine the constants straightforwardly keeping in mind that $\kappa$ depends on $q$ and $\omega$. For the coefficients in the electrolyte we get

$$
\mathcal{C}_{1}=\frac{q V_{0}}{Z e} \frac{\kappa}{q} C_{\text {eff }}(\omega)
$$

and

$$
\mathcal{C}_{2}=-i \frac{\omega}{\omega_{D}} \frac{\kappa}{q} \mathcal{C}_{1}
$$

while for the insulator the coefficients have a similar, but less compact form. Above,

$$
\begin{aligned}
C_{\mathrm{eff}}^{-1}(\omega)= & \left(\kappa \lambda_{D}\right)^{2} \frac{\sinh (q d)}{q d} C_{s}^{-1} \\
& +\kappa \lambda_{D} \frac{q \lambda_{D}\left(q \lambda_{D}+\kappa \lambda_{D}\right)+i \omega / \omega_{D}}{q \lambda_{D}\left(q \lambda_{D}+\kappa \lambda_{D}\right)} \cosh (q d) C_{D}^{-1},
\end{aligned}
$$

which satisfies the definition in Eq. (13a) and reduces to Eq. (13b) in the dc limit. From the general solution for the potential in the electrolyte, Eq. (20), we may now in more detail examine the constraints on $V_{0}$ for the Debye-Hückel approximation to be valid. Straightforward calculations show that $\max \{\phi\} \ll k_{\mathrm{B}} T / Z e$ corresponds to $V_{0} \ll V_{T} \equiv(1$ $\left.+C_{D} / C_{s}\right) k_{\mathrm{B}} T / Z e$ for $q d \ll 1, q \lambda_{D} \ll 1$, and low frequencies.

\section{Long-period and low-frequency modulation}

Next, we consider the regime where the spatial period of the modulation is much longer than all other length scales, i.e., $q \lambda_{D} \ll 1$ and $q d \ll 1$. We also assume that $\omega \ll \omega_{D}$ so that $\kappa \simeq 1 / \lambda_{D}$. In this limit we get

$$
\nu=-\frac{q \sigma_{\infty} V_{0}}{Z e \lambda_{D}} \frac{1}{\omega^{*}+i \omega} e^{-x / \lambda_{D}} \cos (q y) e^{i \omega t}+O\left(\left[q \lambda_{D}\right]^{2}\right)
$$

and

$$
\phi=V_{0} \frac{i \omega}{\omega^{*}+i \omega} e^{-q x} \cos (q y) e^{i \omega t}+O\left(q \lambda_{D}\right),
$$

where we have used the notation of Ajdari [2]: resonance frequency

$$
\omega^{*}=q \lambda_{D}(1+\delta) \omega_{D}
$$

conductivity

$$
\sigma_{\infty}=\left[\sigma^{+}+\sigma^{-}\right]_{\infty}=\epsilon \omega_{D},
$$

and capacitance ratio

$$
\delta=\frac{C_{D}}{C_{s}} .
$$

These results are equivalent to those in Ref. [2] if, similarly to Eq. (13a), we introduce the Debye layer surface charge $\sigma_{D}(y)=Z e \int_{0}^{\infty} d x \nu(x, y)$.

\section{Body force}

Until this point we have used the exponential notation for the temporal dependence. However, since the body force is essentially nonlinear in the electrical potential or density [see the last term in Eq. (3d)] we have to take the real part to get the body force, i.e., $\mathbf{F}=-Z e \nu \nabla \phi=-Z e \operatorname{Re}\{\nu\} \operatorname{Re}\{\boldsymbol{\nabla} \phi\}$ so that we get

$$
\begin{aligned}
\mathbf{F}= & \frac{\eta v_{1}}{\lambda_{D}^{2}} \frac{\cos (2 \omega t+\varphi)}{\omega / \omega^{*}+\omega^{*} / \omega} e^{-x / \lambda_{D}} \times\left[2 \cos ^{2}(q y) \mathbf{e}_{x}+\sin (2 q y) \mathbf{e}_{y}\right] \\
& +O\left(\left[q \lambda_{D}\right]^{2}\right)
\end{aligned}
$$

where following Ref. [2] we have introduced

$$
v_{1} \equiv \frac{q \epsilon V_{0}^{2}}{4 \eta(1+\delta)}
$$

and the frequency-dependent phase shift

$$
\varphi=-\arctan \left(\frac{\omega}{2 \omega^{*}}-\frac{\omega^{*}}{2 \omega}\right) .
$$

In the derivation of Eq. (27a) we have used that

$$
\operatorname{Re}\left\{\frac{e^{i \omega t}}{i \omega+\omega^{*}}\right\} \operatorname{Re}\left\{\frac{i \omega e^{i \omega t}}{i \omega+\omega^{*}}\right\}=\frac{-\cos (2 \omega t+\varphi)}{2 \omega^{*}\left(\omega / \omega^{*}+\omega^{*} / \omega\right)} .
$$

At low frequencies, $\mathbf{F} \propto \omega$, it becomes maximal at the resonance frequency $\omega^{*}$, and then it falls off again at higher 
frequencies. We note that $\lim _{\omega \rightarrow 0} \mathbf{F}=O\left(\left[q \lambda_{D}\right]^{2}\right)$, but this small force will just be balanced by a pressure gradient so that $\lim _{\omega \rightarrow 0} \mathbf{v}=\mathbf{0}$ and $\lim _{\omega \rightarrow 0} \mathbf{i}^{ \pm}=\mathbf{0}$.

\section{E. Linearized flow in quasi-steady-state}

In order to solve the Navier-Stokes equation Eq. (3d), we note that for a body force of small magnitude and with slow temporal variation the fluid response is linear and the flow will approximately be at steady state at each moment in time. We begin by comparing the inertial terms on the left-hand side (LHS) with the viscous term (second term) on the righthand side (RHS). The body force has a characteristic frequency $\omega$ and two characteristic length scales $\lambda_{D}$ and $q^{-1}$ for the $x$ and $y$ directions, respectively. Since $\partial_{t}$ essentially gives a factor of $\omega$, and $\boldsymbol{\nabla}$ essentially gives $\lambda_{D}^{-1} \mathbf{e}_{x}+q \mathbf{e}_{y}$, we can show that the viscous term dominates over the LHS when $\omega \ll \omega_{c}$ where

$$
\omega_{c} \equiv \frac{\eta}{\rho} \min \left\{q^{2}, \lambda_{D}^{-2}\right\}
$$

For $q \lambda_{D} \ll 1$ this means that $\omega_{c}=(\eta / \rho) q^{2}$. In this way, for small Reynolds numbers, we get

$$
\mathbf{0}=-\boldsymbol{\nabla} p+\eta \boldsymbol{\nabla}^{2} \mathbf{v}+\mathbf{F}, \quad \omega \ll \omega_{c}
$$

which is the resulting quasi-steady-flow problem which is linear in the velocity field. Slip-velocity approaches usually rely on this equation (see Ref. [2] and references therein). However, Eqs. (30) and (27a) can actually be solved exactly with a solution given by

$$
\begin{aligned}
\mathbf{v}(\mathbf{r}, t) & =v_{1} \frac{\cos (2 \omega t+\varphi)}{\omega / \omega^{*}+\omega^{*} / \omega} e^{-2 q x}\left[2 q \lambda_{D} \mathcal{G}_{1}\left(\lambda_{D}^{-1}\right) \cos (2 q y) \mathbf{e}_{x}+\mathcal{G}_{1}(2 q) \sin (2 q y) \mathbf{e}_{y}\right] \\
& =v_{1} \frac{\cos (2 \omega t+\varphi)}{\omega / \omega^{*}+\omega^{*} / \omega}\left[-2 q x e^{-2 q x} \cos (2 q y) \mathbf{e}_{x}+\left\{(1-2 q x) e^{-2 q x}-e^{\left.-x / \lambda_{D}\right\}} \sin (2 q y) \mathbf{e}_{y}\right]+O\left(q \lambda_{D}\right),\right. \\
p(\mathbf{r}, t) & =-4 q \eta v_{1} \frac{\cos (2 \omega t+\varphi)}{\omega / \omega^{*}+\omega^{*} / \omega} e^{-2 q x}\left(\frac{1+\mathcal{G}_{2}(2 q)}{\left(1+2 q \lambda_{D}\right)^{2}} \cos (2 q y)+\mathcal{G}_{2}(0)\right) \\
& =-4 q \eta v_{1} \frac{\cos (2 \omega t+\varphi)}{\omega / \omega^{*}+\omega^{*} / \omega}\left(e^{-2 q x} \cos (2 q y)+\frac{e^{-x / \lambda_{D}}}{4 q \lambda_{D}}[1+\cos (2 q y)]\right)+O\left(q \lambda_{D}\right),
\end{aligned}
$$

as may be verified by direct insertion. Above,

$$
\mathcal{G}_{1}(k)=\frac{1-2 q \lambda_{D}}{\left[1-\left(2 q \lambda_{D}\right)^{2}\right]^{2}}\left[1-e^{-\left(\lambda_{D}^{-1}-2 q\right) x}-\left(1-2 q \lambda_{D}\right) k x\right]
$$

and

$$
\mathcal{G}_{2}(k)=\frac{1+k \lambda_{D}}{4 q \lambda_{D}} e^{-\left(\lambda_{D}^{-1}-2 q\right) x}
$$

have been introduced. As seen the flow decays exponentially over a length scale of $1 / q$ when $\omega \ll \omega_{c}$. When the frequency becomes comparable to or larger than $\omega_{c}$ we have competing length scales since the $\partial_{t} \mathbf{v}$ term introduces an additional length scale $(\eta / \rho \omega)^{1 / 2}$, which as mentioned becomes $\left(\omega_{c} / \omega\right)^{1 / 2}(1 / q)$ for $q \lambda_{D} \ll 1$. So in the above expressions for $\mathbf{v}$ and $p$ we expect that the spatial cutoff length changes from $(2 q)^{-1}$ to $\Lambda$ with

$$
\Lambda(\omega) \sim \frac{1}{2 q} \min \left\{1, \sqrt{\omega_{c} / \omega}\right\}
$$

Even for $\omega \ll \omega^{*}$ the condition $\omega \ll \omega_{c}$ is not necessarily satisfied. In fact, for the numbers in Table I we have $\omega_{c}<\omega^{*}$ $<\omega_{D}$ so at resonance $2 q \Lambda\left(\omega^{*}\right)=\sqrt{\omega_{c} / \omega^{*}} \ll 1$.

\section{F. Flow and separation of length scales}

As mentioned above the flow is typically analyzed by slip-velocity approaches and here we show how such an approach gives an asymptotic solution in full agreement with the exact solution. We study the flow over a $\lambda_{D}$ scale at the boundary first and then a $q^{-1}$ scale. For this boundary layer approach, we assume that for $x \leqslant 3 \lambda_{D}$, we have $v_{x} \approx 0$. Solving for the pressure and substituting into the $y$ component of Eq. (30) we get

$$
v_{y}=v_{s}(y, t)\left(1-e^{-x / \lambda_{D}}\right)+O\left(q \lambda_{D}\right), \quad x \lesssim 3 \lambda_{D},
$$

with the prefactor 

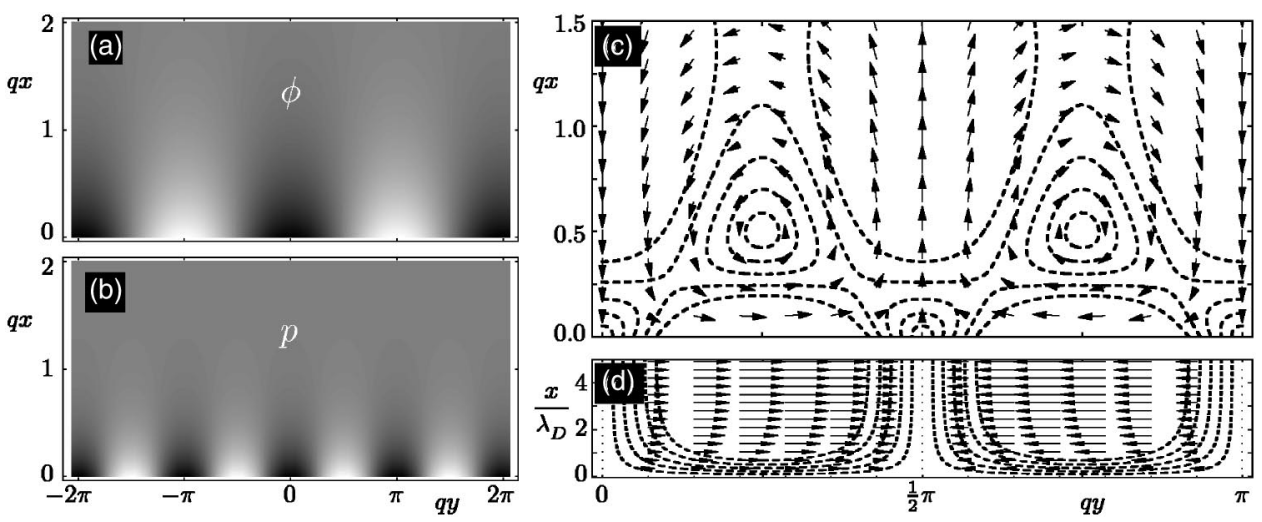

FIG. 2. The potential $\phi$, pressure $p$, and velocity field $\mathbf{v}$. (a) shows a gray scale plot of the amplitude of the potential $\phi$ as a function of $q x$ and $q y$, Eq. (25), and (b) the pressure $p$, Eq. (39). Notice the period doubling in the pressure compared to the electric potential. (c) shows a snapshot of the harmonically oscillating velocity field $\mathbf{v}$ in the bulk, Eq. (38), and (d) likewise in the Debye layer, Eq. (35). The flow pattern contains rolls, which are indicated by contours of constant velocity (dashed lines).

$$
v_{s}(y, t) \equiv v_{1} \frac{\cos (2 \omega t+\varphi)}{\omega / \omega^{*}+\omega^{*} / \omega} \sin (2 q y) .
$$

In the limit $x \gtrsim 3 \lambda_{D}$ and $q \lambda_{D} \ll 1$ the velocity $v_{s}$ can be interpreted as a slip velocity at the wall acting as a conveyor belt for the bulk fluid [see Fig. 2(d)].

For $x \gtrsim 3 \lambda_{D}$ we have that $\mathbf{F}$ is exponentially suppressed and we solve Eq. (30) together with Eq. (3e) and the boundary condition

$$
\mathbf{v}(\mathbf{r}, t)_{x=0}=v_{s}(y, t) \mathbf{e}_{y} .
$$

To lowest order in $q \lambda_{D}$ this gives

$$
\begin{aligned}
\mathbf{v} \simeq & v_{1} \frac{\cos (2 \omega t+\varphi)}{\omega / \omega^{*}+\omega^{*} / \omega} e^{-2 q x}\left[-2 q x \cos (2 q y) \mathbf{e}_{x}\right. \\
& \left.+(1-2 q x) \sin (2 q y) \mathbf{e}_{y}\right],
\end{aligned}
$$

and

$$
p \simeq-4 q \eta v_{1} \frac{\cos (2 \omega t+\varphi)}{\omega / \omega^{*}+\omega^{*} / \omega} e^{-2 q x} \cos (2 q y)
$$

If we now substitute into Eq. (3d) we get (RHS-LHS) $\propto e^{-x / \lambda_{D}}+O\left(\omega / \omega_{D}\right)+O\left(\left[q \lambda_{D}\right]^{2}\right)$ which shows that Eqs. (38) and (39) are indeed excellent approximations to the full solution of the nonlinear time-dependent Navier-Stokes equation Eq. (3d) for $x \gtrsim 3 \lambda_{D}$. For the incompressibility constraint Eq. (3e), our solution gives $\boldsymbol{\nabla} \cdot \mathbf{v}=O\left(\left[q \lambda_{D}\right]^{2}\right)$. In Fig. 2(c) we show a plot of the velocity field, Eq. (38), along with the contours for constant velocity.

We note that in the limit $x \gtrsim 3 \lambda_{D}$ the exact solutions, Eqs. (31b) and (32b) reduce to Eqs. (38) and (39) for the bulk.

\section{FULL NUMERICAL SOLUTION}

In this section we present results from numerical finite element simulations (FEMLAB) of the five coupled equations Eqs. (3a)-(3e), with the boundary conditions in Eqs. (4a), (4b), and (5)-(7). For simplicity we assume a low Reynolds number so that we can neglect the inertial term $(\mathbf{v} \cdot \boldsymbol{\nabla}) \mathbf{v}$ in Eq. (3d). This provides full access to the temporal and spatial evolution of the physical quantities $n^{ \pm}, \phi, \mathbf{i}^{ \pm}, \mathbf{v}$, and $p$. The spatially periodic problem (in the $y$ direction) would typi- cally be handled by applying periodic boundary conditions to the unit cell (e.g., $0<y<2 \pi / q$ ). However, due to the symmetry of $V_{\text {ext }}$ the computational domain can be reduced to $0<y<\pi / q$ with homogeneous Dirichlet or Neumann boundary conditions. For the $x$ direction our domain is cut off at a distance $x=6 \pi / q$ from the interface using Dirichlet boundary conditions for the fields. Near the interface to the insulator, $0<x \leqq 3 \lambda_{D}$, we employ a structured grid to resolve the Debye layer. For the temporal evolution we employ the FEMLAB time stepper directly starting from initial solutions at $t=0$ which are zero everywhere. The duration of the transient depends on the inertia in the system, but typically the temporal harmonic state is fully evolved after a time of the order $(10-100) \omega^{-1}$. For our simulations we have used the typical values in Table I except for the modulation where we have used $q^{-1}=10^{-6} \mathrm{~m}$ and consequently $\omega^{*}=\omega_{c}=10^{6} \mathrm{rad} / \mathrm{s}$. For the external potential $V_{\text {ext }}$ we have used the imaginary part of Eq. (1) (which is zero at $t=0$ ) with an amplitude $0<V_{0}$ $<10 \mathrm{~V}$ and a driving frequency $10^{4}<\omega<10^{8} \mathrm{rad} / \mathrm{s}$.

In order to directly compare our numerical results to the linearized theory we normalize frequencies by the resonance frequency $\omega^{*}$, velocities by $v_{1}$, voltages by the thermal voltage $V_{T}$, and capacitances by $C_{0}$.

Figure 3 shows numerical results for the effective capacitance $C_{\text {eff }}$ [see definition in Eq. (13a)] as a function of the frequency for varying amplitudes $V_{0}$ of the external voltage. The dashed line shows the corresponding analytical result from Eq. (23c). As seen there is a good agreement between numerical results and our analytical predictions even for $V_{0}$ $>V_{T}$ where the Debye-Hückel approximation is typically expected to work poorly. We furthermore note that at low frequencies $\operatorname{Re}\left\{C_{\text {eff }}\right\}$ approaches $C_{0}$ in full agreement with the analytics and the log-log plot also reveals two distinct regimes for $\omega<\omega^{*}$ and $\omega>\omega^{*}$. In fact, dissipation is maximal exactly at the resonance frequency $\omega^{*}$ predicted by the linear theory. The inset shows the relative error of the DebyeHückel approximation which at large voltages saturates at a value of the order $(1+\delta)^{-1}$, here equal to 0.09 .

The linear theory predicts a harmonic velocity field with a vanishing time average and our numerical simulations confirm this low-frequency dynamics $\left[\right.$ see $\max _{\mathbf{r}, t}\{v(\mathbf{r}, t)\}$ in Fig. 4(a)]. The corresponding solid line shows exact results within the Debye-Hückel approximation [20] and the dashed 


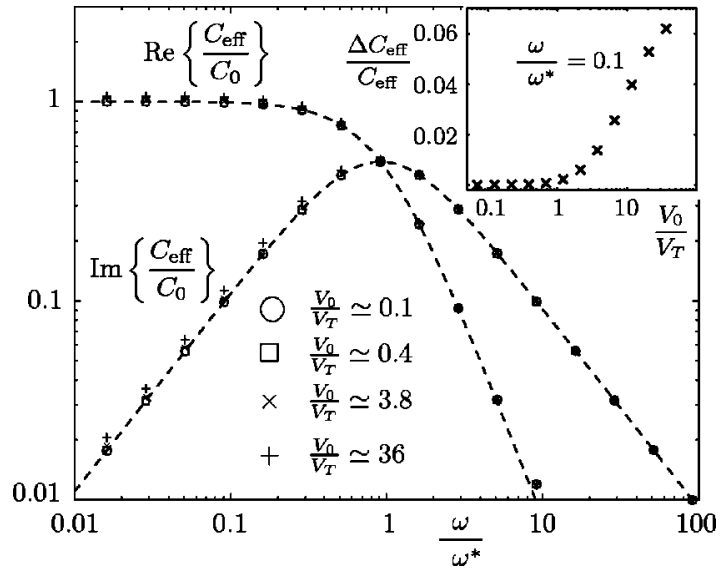

FIG. 3. The real and the imaginary parts of the normalized effective capacitance $C_{\text {eff }}\left(V_{0}, \omega\right) / C_{0}$ versus the normalized frequency $\omega / \omega^{*}$ for four different values of the voltage amplitude $V_{0} / V_{T}$. The dashed lines show the analytical results of the linear theory, Eq. (23c). The inset shows the relative deviation (below resonance) of the full numerical solution for $C_{\text {eff }}\left(V_{0}\right) / C_{0}$ from the Debye-Hückel result as a function of $V_{0} / V_{T}$.

line shows $\left(\omega / \omega^{*}+\omega^{*} / \omega\right)^{-1}$ as suggested by Eq. (38). As expected the induced harmonic motion peaks at the resonance frequency $\omega^{*}$ with a characteristic speed $v_{1}$ [Eq. (27b)]. However, in the high-frequency dynamics we observe the coexistence of a small but nonvanishing time-averaged component, $0<\max _{\mathbf{r}}\left\{\langle v(\mathbf{r}, t)\rangle_{t}\right\} \ll v_{1}$. Figure 4(a) shows $\max _{\mathbf{r}}\left\{\langle v(\mathbf{r}, t)\rangle_{t}\right\}$ as a function of frequency for different external voltages. The corresponding solid line shows exact results within the Debye-Hückel approximation [20]. Figure 4(b) shows a particular example of the time-averaged velocity field $\langle\mathbf{v}(\mathbf{r}, t)\rangle_{t}$.

\section{DISCUSSION}

We have analyzed the full nonequilibrium electrohydrodynamics of the Debye screening layer that arise in an aqueous binary solution near a planar wall when applying a spatially modulated ac voltage $V_{0} \cos (q y) e^{i \omega t}$ [Eq. (1)]. Using first order perturbation theory we have obtained analytic solutions for the pressure and velocity fields of the electrolyte and for the electric potential. Our analytical solution applies to the low-frequency Debye-Hückel regime where the amplitude $V_{0}$ of the external potential is lower than the thermal voltage $V_{T}$ and the driving frequency $\omega$ is lower than the inverse response time of the electrolyte $\omega_{D}=\sigma_{\infty} / \epsilon$ (see Secs. IV A and IV C). It should be noted that our analysis does not cover the special case of suddenly applied step voltages, where the system selects its own intrinsic time scale different from the external time scale $1 / \omega[19]$.

Furthermore, we have limited ourselves to the diffusive regime where convection can be neglected corresponding to a sufficiently low driving amplitude, $V_{0} \ll V_{c}$ where $V_{c}$ $\equiv \sqrt{(1+\delta) \eta D / \epsilon}$ is a convective voltage (see first paragraph of Sec. IV B; $\mathbf{v} \sim v_{1} \mathbf{e}_{y}$ in the Debye layer). We have also considered the low-frequency regime $\omega \ll \omega_{c}$ where viscosity dominates over inertia (see Sec. IV E).

Finally, we have considered the limit with the spatial modulation being much longer than all other length scales in the problem, i.e., $q d \ll 1$ and $q \lambda_{D} \ll 1$ (see Sec. IV C). In summary this means that the analytical studies of the effect of Eq. (1) are valid in the limits

$$
\begin{aligned}
& q \ll \min \left\{d^{-1}, \lambda_{D}^{-1}\right\}, \\
& \omega \ll \min \left\{\omega_{D}, \omega_{c}\right\}, \\
& V_{0} \ll \min \left\{V_{T}, V_{c}\right\} .
\end{aligned}
$$

As a main result we have supplied a proof for the validity of the capacitor model. The full dynamics seems, however, not to be captured by the capacitor model. Taking the time average in Eqs. (27a) and (38) we get $\langle\mathbf{F}\rangle_{t}=\mathbf{0}$ and $\langle\mathbf{v}\rangle_{t}=\mathbf{0}$ (in full agreement with the discussion in Ref. [2]). In contrast, we obtain $\langle\mathbf{F}\rangle_{t} \neq \mathbf{0}$ if we begin from Eqs. (18a) and (20) and without expanding in $\omega / \omega_{D}$ and $q \lambda_{D}$, the result being finite even in the zero-frequency limit. Somewhat similar results were reported in another nonequilibrium study [5], though for a different geometry. Naively, this observation could suggest that $\langle\mathbf{v}\rangle_{t} \neq \mathbf{0}$ contrary to the statement in Ref. [2]. How-
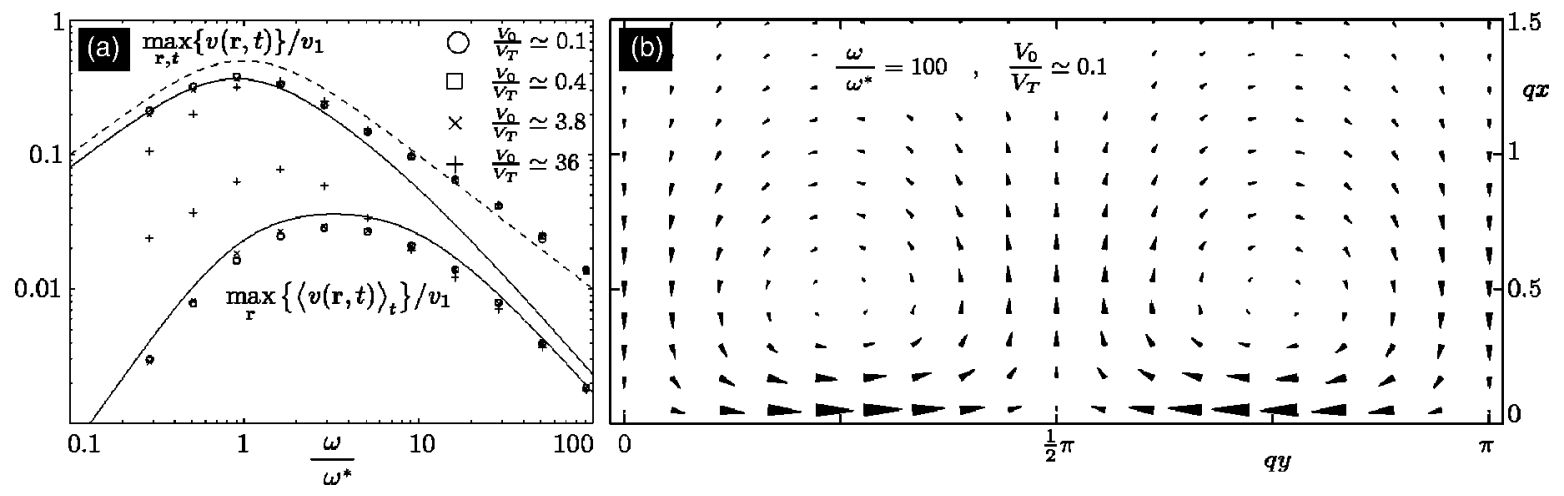

FIG. 4. Numerical simulations of flow. (a) shows the maximal velocity of the harmonic flow $\max _{\mathbf{r}, t}\{v(\mathbf{r}, t)\}$ and the maximal velocity of the time-averaged flow $\max _{\mathbf{r}}\left\{\langle v(\mathbf{r}, t)\rangle_{t}\right\}$, both versus normalized frequency. The solid lines show exact results within the Debye-Hückel approximation [20] and the dashed line shows $\left(\omega / \omega^{*}+\omega^{*} / \omega\right)^{-1}$ as suggested by Eq. (38). (b) shows an example of the time-averaged velocity field $\langle\mathbf{v}(\mathbf{r}, t)\rangle_{t}$. 
ever, by also averaging over the $y$ direction we get $\left\langle F_{y}\right\rangle_{t, y}$ $=0$ suggesting that $\left\langle v_{y}\right\rangle_{t, y}=0$ in agreement with the symmetry arguments emphasized in Ref. [2]. If the finite $\langle\mathbf{F}\rangle_{t}$ does not give the fluid a directional flow globally, we might speculate that, at high frequencies, it makes the fluid circulate in nonoscillating vortices [see Fig. 4(a)], whereas the fluid is at rest at zero frequency, despite $\langle\mathbf{F}\rangle_{t}$ being finite. The solution to this apparent contradiction lies in the pressure, which will compensate the body force at low frequencies. We can explicitly show that the time-averaged body force can be written as

$$
\langle\mathbf{F}(\mathbf{r}, t)\rangle_{t}=\nabla p_{\mathbf{F}}(\mathbf{r})+O\left(\left[\omega / \omega_{D}\right]^{2}\right)
$$

where

$$
\begin{aligned}
p_{\mathbf{F}}(\mathbf{r}) \equiv & \frac{\frac{1}{4} \epsilon q^{2} V_{0}^{2}}{\left[q \lambda_{D} \cosh (q d)+\left(\epsilon / \epsilon_{s}\right) \sqrt{1+\left(q \lambda_{D}\right)^{2}} \sinh (q d)\right]^{2}} \\
& \times e^{-2 \sqrt{1+\left(q \lambda_{D}\right)^{2}} x / \lambda_{D}} \cos ^{2}(q y), \\
= & q \eta v_{1}\left(1+\frac{C_{D}}{C_{s}}\right)\left(\frac{C_{\mathrm{eff}}(0)}{C_{D}}\right)^{2} \frac{1+\left(q \lambda_{D}\right)^{2}}{\left(q \lambda_{D}\right)^{2}} \\
& \times e^{-2 \sqrt{1+\left(q \lambda_{D}\right)^{2}} x / \lambda_{D}} \cos ^{2}(q y) .
\end{aligned}
$$

The form of Eq. (41) suggests that

$$
\langle\mathbf{v}(\mathbf{r}, t)\rangle_{t}=\mathbf{0}+O\left(\left[\omega / \omega_{D}\right]^{2}\right)
$$

with $p_{\mathbf{F}}$ being a pressure that compensates the low-frequency part of the body force [see Eq. (30)]. The time-averaged velocity field $\langle\mathbf{v}(\mathbf{r}, t)\rangle_{t}$ can be calculated rigorously and the complex expression (not shown) agrees fully with Eq. (43). At high frequencies we expect stationary vortices, Fig. 4(b), to coexist with the harmonic fluid motion illustrated in Fig. 2(c), whereas at low frequencies the circulation vanishes and we are left with the pure harmonic motion. Our timedependent finite-element simulations in Fig. 4 support this picture, and similar time-averaged flow in a slightly different geometry has been observed both experimentally, theoretically, and numerically [4-6].

\section{CONCLUSION}

Our results provide the theoretical underpinning of the capacitor model widely used in the literature $[2-4,6,7,15,19]$, and form a firm starting point for future studies of electrokinetic pumps and mixers driven by spatially modulated surface potentials. In general for large values of $\delta$ we find that the Debye-Hückel approximation works well even at elevated voltages [agreement within less than $(1+\delta)^{-1}$ ] where it is typically expected to work poorly. However, our nonequilibrium approach has also revealed interesting shortcomings in the capacitor approach for high-frequency dynamics where static vortices appear along with the harmonic rolls also predicted by the capacitor model.

\section{ACKNOWLEDGMENTS}

We thank A. Ajdari for stimulating discussions and T. S. Hansen for sharing initial numerical results with us. N.A.M. is supported by the Danish Technical Research Council (Grant No. 26-03-0073) and L.B. by a Socrates/Erasmus grant from the European Community.
[1] S. R. Yeh, M. Seul, and B. I. Shraiman, Nature (London) 386, 57 (1997).

[2] A. Ajdari, Phys. Rev. E 61, R45 (2000).

[3] A. B. D. Brown, C. G. Smith, and A. R. Rennie, Phys. Rev. E 63, 016305 (2000).

[4] N. G. Green, A. Ramos, A. González, H. Morgan, and A. Castellanos, Phys. Rev. E 61, 4011 (2000).

[5] A. González, A. Ramos, N. G. Green, A. Castellanos, and H. Morgan, Phys. Rev. E 61, 4019 (2000).

[6] N. G. Green, A. Ramos, A. González, H. Morgan, and A. Castellanos, Phys. Rev. E 66, 026305 (2002).

[7] F. Nadal, F. Argoul, P. Kestener, B. Pouligny, C. Ybert, and A. Ajdari, Eur. Phys. J. E 9, 387 (2002).

[8] A. Ajdari, Phys. Rev. E 65, 016301 (2002).

[9] I. Gitlin, A. D. Stroock, G. M. Whitesides, and A. Ajdari, Appl. Phys. Lett. 83, 1486 (2003).

[10] V. Studer, A. Pépin, Y. Chen, and A. Ajdari, Analyst (Cambridge, U.K.) 129, 944 (2004).

[11] B. P. Cahill, L. J. Heyderman, J. Gobrecht, and A. Stemmer, Phys. Rev. E 70, 036305 (2004).

[12] M. Arnoldus and M. Hansen, BS thesis, Department of Micro and Nanotechnology, Technical University of Denmark, 2004 (unpublished).
[13] T. S. Hansen, MS thesis, Department of Chemical Engineering, Technical University of Denmark, 2004 (unpublished).

[14] A. Ramos, H. Morgan, N. G. Green, and A. Castellanos, J. Phys. D 31, 2338 (1998).

[15] H. Morgan and N. G. Green, AC Electrokinetics: Colloids and Nanoparticles, Microtechnologies and Microsystems Series (Institute of Physics Publishing, Bristol, 2003).

[16] H. A. Stone, A. D. Stroock, and A. Ajdari, Annu. Rev. Fluid Mech. 36, 381 (2004).

[17] M. Z. Bazant and T. M. Squires, Phys. Rev. Lett. 92, 066101 (2004).

[18] T. M. Squires and M. Z. Bazant, J. Fluid Mech. 509, 217 (2004).

[19] M. Z. Bazant, K. Thornton, and A. Ajdari, Phys. Rev. E 70, 021506 (2004)

[20] The very long analytical expression is not displayed. We assume a low Péclet number and employ the Debye-Hückel approximation to linearize the electrodynamics and calculate the full expression for $\mathbf{F}$ without expansions in $\omega / \omega_{D}, q \lambda_{D}$, and $q d$. Subsequently we solve the hydrodynamics in the lowReynolds-number limit, but with the $\partial_{t} \mathbf{v}$ term taken into account. 